\title{
CARGAS FÍSICAS E PSÍQUICAS NO TRABALHO DE AGENTES COMUNITÁRIOS DE SAÚDE
}

Maria Saleti Vogt ${ }^{1}$, Carmem Lúcia Colomé Beck², Francine Cassol Prestes ${ }^{3}$, Paola da Silva Diaz ${ }^{4}$, Juliana Petri Tavares ${ }^{5}$, Gilson Mafaccioli da Silva ${ }^{6}$

\begin{abstract}
RESUMO: Trata-se de uma pesquisa exploratório-descritiva, com abordagem qualitativa em que se aplicou um questionário para a coleta dos dados a 8 agentes comunitários de saúde, no ano de 2009, com o objetivo de identificar as cargas de trabalho físicas e psíquicas. Para a análise dos dados utilizou-se a técnica da análise de conteúdo temática. No que tange às cargas físicas a categoria identificada foi condições de trabalho inadequadas com destaque ao ambiente laboral inadequado e precariedade dos equipamentos de trabalho. Em relação às cargas psíquicas, as categorias identificadas foram falta de reconhecimento por parte de alguns integrantes da comunidades; incapacidade de resolver problemas e dificuldades de relacionamento interpessoal com a comunidade e equipe de saúde da unidade. Esse estudo aponta a necessidade de traçar estratégias de intervenção junto aos agentes e serviço de saúde para promover ações que contribuam para a melhoria da qualidade de vida e saúde destes trabalhadores.

PALAVRAS-CHAVE: Carga de trabalho; Saúde do trabalhador; Equipe de assistência ao paciente.

\section{PHYSICAL AND PSYCHOLOGICAL BURDENS IN THE WORK OF COMMUNITY HEALTH ASSISTANTS}

ABSTRACT: Exploratory-descriptive research from 2009, with a qualitative approach in which a questionnaire for collecting data was applied to 8 community health assistants, with the aim of identifying the physical and psychological burdens resulting from the work. Thematic content analysis was used for analysis of the data. Regarding physical burdens, the category identified was inappropriate work conditions, with emphasis on an inappropriate work environment and inadequacies regarding work equipment. In relation to psychological burdens, the categories identified were lack of recognition on the part of some members of communities; inability to resolve interpersonal relationship problems and difficulties with the community and health unit team. This study indicates the need to draw up intervention strategies in collaboration with the health assistants and health service so as to promote actions which contribute to the improvement of quality of life and health among these workers.

KEYWORDS: Workload; Worker's health; Patient care team.

\section{CARGAS FÍSICAS Y PSÍQUICAS EN EL TRABAJO DE AGENTES COMUNITARIOS DE SALUD}

RESUMEN: Esta es una investigación exploratoria descriptiva, con abordaje cualitativo en que se ha aplicado un cuestionario para recoger datos de ocho agentes comunitarios de salud, en el año de 2009, con el objetivo de identificar las cargas de trabajo físicas y psíquicas. Para el análisis de los datos, fue utilizada la técnica del análisis de contenido temática. Acerca de las cargas físicas, la categoría identificada fue: condiciones de trabajo inadecuadas con destaque al ambiente laboral inadecuado y precariedad de los equipos de trabajo. Acerca de las cargas psíquicas, las categorías identificadas fueron: falta de reconocimiento por parte de algunos integrantes de la comunidad; incapacidad de resolver problemas y dificultades de relación interpersonal con la comunidad y equipo de salud de la unidad. Ese estudio indica la necesidad de pensar estrategias de intervención junto a los agentes y servicio de salud para promover acciones que contribuyan para mejorar la cualidad de vida y salud de estes trabajadores. PALABRAS CLAVE: Carga de trabajo; Salud del trabajador; Equipo de asistencia al paciente.

\footnotetext{
${ }^{1}$ Fisioterapeuta. Doutora em Fisioterapia. Professora do Departamento de Fisioterapia e Reabilitação da Universidade Federal de Santa Maria - UFSM.

${ }^{2}$ Enfermeira. Doutora em Enfermagem. Professora do Departamento de Enfermagem da UFSM. Membro do Grupo de Pesquisa Trabalho, Saúde, Educação e Enfermagem.

${ }^{3}$ Enfermeira. Mestre em Enfermagem. Membro do Grupo de Pesquisas Trabalho, Saúde, Educação e Enfermagem.

${ }^{4}$ Enfermeira. Mestranda pelo Programa de Pós-Graduação em Enfermagem da UFSM. Membro do Grupo de Pesquisas Trabalho, Saúde, Educação e Enfermagem.

${ }^{5}$ Enfermeira. Doutoranda pelo Programa de Pós-Graduação em Enfermagem da UFSM. Membro do Grupo de Pesquisa Trabalho, Saúde, Educação e Enfermagem.

${ }^{6}$ Médico Psiquiatra. Mestre em Psicologia. Membro do Grupo de Pesquisa Trabalho, Saúde, Educação e Enfermagem.
}

Autor correspondente:

Recebido: 13/09/2011

Francine Cassol Prestes

Aprovado: 29/05/2012

Universidade Federal de Santa Maria

Rua Tuiuti, 520 - 97015-660 - Santa Maria-RS-Brasil

E-mail: francinecassol@gmail.com 


\section{INTRODUÇÃO}

O Programa de Agentes Comunitários (PACS) existe desde o início dos anos 90, quando se iniciou o processo de consolidação da descentralização de recursos no âmbito do Sistema Único de Saúde (SUS). O PACS foi inspirado em experiências de prevenção de doenças por meio de informações e orientações sobre cuidados de saúde ${ }^{(1)}$.

O desenvolvimento das principais ações desse programa se dá por meio dos Agentes Comunitários de Saúde (ACS), pessoas escolhidas dentro da própria comunidade para atuarem junto à população $\mathrm{O}^{(1)}$. A profissão de ACS está amparada pela Lei n. 11.350, com a função de exercer, sob supervisão, atividades de prevenção de doenças e promoção da saúde, por meio de ações domiciliares ou comunitárias, individuais ou coletivas, desenvolvidas de acordo com as diretrizes do Sistema Único de Saúde (SUS) ${ }^{(2)}$.

O trabalho do ACS requer uma série de conhecimentos referentes à área da saúde que muitas vezes são desconhecidos por estes, pelo fato de não possuírem formação profissional. Assim, o ACS assume uma nova responsabilidade ao compor a equipe de saúde da família, necessitando conhecer certos conteúdos e práticas referentes à biomedicina ${ }^{(3)}$.

Além disso, o ACS por vivenciar os problemas e morar na comunidade em que desempenha seu trabalho, figura como importante elo de interlocução entre a equipe de saúde e os usuários. Este fato define um envolvimento pessoal diferenciado com questões relacionadas à saúde das famílias acompanhadas por ele ${ }^{(4)}$. Assim, as situações vivenciadas no seu ambiente de trabalho suscitam sentimentos intensos e contraditórios ${ }^{(5)}$.

$\mathrm{O}$ movimento de cuidado à saúde do trabalhador decorre do crescente adoecimento desta parcela populacional, na qual estão inseridos os que cuidam da saúde da população sendo que estes, por vezes, esquecem de cuidar da própria saúde e das condições de seu ambiente de trabalho ${ }^{(6)}$.

As cargas de trabalho são conceituadas como o conjunto de esforços desenvolvidos para atender às exigências das tarefas, abrangendo os esforços físicos, cognitivos e os psicoafetivos ${ }^{(7)}$. Conceitualmente, as cargas são divididas em dois grupos: aquelas que têm materialidade externa ao corpo do trabalhador, como as cargas físicas, químicas, biológicas e mecânicas, e as que só adquirem materialidade na corporeidade humana, ou seja, as cargas fisiológicas e psíquicas ${ }^{(8)}$. Neste estudo, optou-se por investigar as cargas físicas e psíquicas no trabalho dos ACS.
As cargas físicas podem ser representadas por ruídos, vibrações e a umidade, que ocasionam alterações nos mecanismos fisiológicos humanos, podendo ou não ser reversíveis. Já as cargas psíquicas, podem ser provocadas por situações de tensão prolongada como atenção constante, ritmo de trabalho acelerado, trabalho perigoso, supervisão constante, ou por outras situações, o que limita o uso da capacidade mental decorrente de atividades desprovidas de conteúdo, da separação entre concepção e execução do trabalho, hierarquia, ocasionando a desqualificação do trabalhador ${ }^{(8)}$.

Neste contexto, as cargas de trabalho físicas e psíquicas a que estão expostos os ACS se constituem em uma temática que necessita ser investigada, tendo em vista a necessidade de se conhecer os fatores intervenientes na saúde destes trabalhadores.

Diante destas questões, foi realizada uma pesquisa junto a Agentes Comunitários de uma Unidade de Saúde do Estado do Rio Grande do Sul/Brasil, com o objetivo de identificar as cargas de trabalho físicas e psíquicas a que estes trabalhadores estão submetidos.

Portanto, justifica-se este estudo pela intenção de conhecer as cargas de trabalho a que os ACS estão expostos e, a partir daí, traçar estratégias adequadas de intervenção junto aos agentes e ao serviço de saúde, no sentido de promover ações que contribuam para a melhoria da qualidade de vida e saúde destes trabalhadores, bem como a qualidade do atendimento por eles prestado.

\section{MÉTODO}

O presente estudo se caracteriza como uma pesquisa exploratório-descritiva, com abordagem qualitativa. Esta pesquisa foi realizada em uma equipe de PACS de uma Unidade Básica de Saúde (UBS) de um município do estado do Rio Grande do Sul. A escolha dos ACS desta equipe se deu pelo fato dos pesquisadores desenvolverem aulas práticas com acadêmicos de enfermagem nesta UBS. Além disso, os agentes que atuavam nesta unidade tinham boa aderência e receptividade a propostas como esta.

A população de agentes desta unidade é composta por onze trabalhadores. Os critérios de inclusão dos sujeitos do estudo foram: ser ACS da referida UBS, e estar em exercício da profissão. Foram excluídos aqueles que estavam em licença de trabalho por motivos diversos no período de coleta dos dados, sendo que participaram desta pesquisa 8 ACS.

$\mathrm{O}$ instrumento de coleta de dados utilizado foi um questionário, incluindo questões abertas e fecha- 
das. Na primeira parte estavam contidos os dados sociodemográficos e na segunda questões específicas sobre o tema proposto nesta pesquisa. Foi realizado um estudo-piloto com três ACS no intuito de validar o referido instrumento; estes participantes foram incluídos no estudo, uma vez que o instrumento não necessitou de alterações.

Participaram da coleta de dados, além dos docentes pesquisadores, dois acadêmicos do curso de graduação em Enfermagem, os quais receberam capacitação específica para esta atividade, o que possibilitou a clareza dos dados a serem pesquisados. A coleta de dados foi realizada mediante agendamento de datas e horários com os participantes, sendo que o local estabelecido pelos ACS foi a UBS, no horário de trabalho e de acordo com a disponibilidade dos agentes.

Para a análise dos dados utilizou-se a técnica da análise de conteúdo temática ${ }^{(9)}$, que se constitui de três etapas: ordenação, classificação dos dados e análise final. A análise dos dados foi realizada em torno de dois eixos temáticos: cargas físicas e cargas psíquicas no trabalho do ACS.

Para a garantia da confidencialidade dos dados dos ACS envolvidos no estudo, os questionários foram codificados com números arábicos, de um a oito, os quais foram numerados aleatoriamente. Os participantes leram e assinaram o Termo de Consentimento Livre e Esclarecido, após a elucidação e apresentação da proposta de estudo a qual respeitou a Resolução 196/96 do Conselho Nacional de Saúde. Este projeto tramitou nos órgãos competentes e obteve parecer favorável do Comitê de Ética em Pesquisa da Universidade Federal de Santa Maria, protocolado sob o CAAE de número 0221.0.243.000-08.

\section{RESULTADOS}

No que tange aos dados sociodemográficos, destaca-se que todas as ACS são do sexo feminino. A idade das participantes variou de 25 a 40 anos, com distribuição uniforme neste intervalo. No que se refere ao tempo de atividade laboral, as agentes possuem de 4 a 12 anos de trabalho como ACS. Quando perguntado se possuíam outra atividade remunerada, todas referiram que não, mas destacaram a realização de atividades domésticas.

Em relação a gostar da atividade que desenvolvem como ACS, todas responderam afirmativamente, pois têm a oportunidade de ajudar e esclarecer as pessoas, especialmente as menos favorecidas, levar alegria as pessoas e trocar conhecimentos com os usuários.
Além do levantamento dos dados sociodemográficos, as ACS foram perguntadas acerca de alguns hábitos de vida, no sentido dos mesmos interferirem na sua condição de saúde. Assim, foram questionadas quanto à prática de exercícios físicos, ao uso do tabaco, a presença de sinais e sintomas de cargas físicas e psíquicas no cotidiano, bem como da realização de tratamento ou acompanhamento regular do seu estado de saúde.

Identificou-se que as ACS não praticavam exercícios físicos regularmente, uma agente respondeu que fuma há 25 anos, uma média de 10 cigarros por dia. Todas as ACS relataram sentir dores nas pernas, coluna vertebral e cansaço. Em relação à realização de tratamento ou acompanhamento regular de saúde, uma ACS respondeu fazer tratamento para hipotireoidismo, depressão e ansiedade e as demais responderam não fazer nenhum tipo acompanhamento ou tratamento. Quatro agentes reconhecem que o trabalho tem influenciado em seu adoecimento.

Com relação às cargas físicas, emergiu a categoria Condições de trabalho inadequadas. Nesta categoria, as ACS apontaram o ambiente laboral inadequado, bem como a precariedade dos equipamentos de trabalho. Quanto ao ambiente laboral, este foi caracterizado pela unidade de saúde a que as agentes estão vinculadas; pelas ruas da comunidade em que transitam e pelas moradias que visitam. As ACS identificaram como elementos que lhe causam algum tipo de incômodo os ruídos, o excesso de frio ou de calor, a presença de odores, a ventilação inadequada das moradias, a poeira das ruas e a presença de animais domésticos nas moradias.

Em relação aos equipamentos de trabalho, as ACS referiram não serem adequados para a realização da atividade laboral, destacando a falta de uniforme; a carência de materiais para medir e pesar as crianças; a falta de protetor solar e de bolsas para transportar seus materiais de trabalho.

No que tange às cargas psíquicas, as categorias identificadas foram Falta de reconhecimento por parte de alguns integrantes da comunidade; Incapacidade de resolver certos problemas e dificuldade de relacionamento interpessoal com a comunidade e equipe de saúde da unidade. Com relação à falta de reconhecimento por parte de alguns integrantes da comunidade, acredita-se que esta experiência pode refletir negativamente na saúde do trabalhador. Uma agente assim se expressou:

Acho que não sou reconhecida pelo meu trabalho, pois posso fazer dez acertos no trabalho, mas se falhar em um sou muito cobrada pela comunidade. (ACS 8) 
Por outro lado, outra agente relatou a satisfação vivenciada diante do reconhecimento de seu trabalho, expresso pela acolhida que recebem nas residências, como evidencia o fragmento a seguir:

Eu adoro poder chegar numa casa e ser bem recebida, até porque eles estão sempre carentes, pessoas que moram sozinhas principalmente. (ACS 2)

As agentes também mencionaram como carga psíquica a incapacidade de resolver alguns problemas, uma vez que atuam em grandes áreas geográficas, com inúmeras famílias e, por conseguinte, lidam com a complexidade na saúde, nas relações, no processo de viver, adoecer e morrer.

Outra carga psíquica no trabalho das ACS foram as dificuldades no relacionamento interpessoal com a comunidade e equipe de saúde da unidade. Os fragmentos a seguir exemplificam a categoria:

Com algumas exceções, nem todos nos tratam bem, nem sempre estão satisfeitos com o nosso trabalho, sempre querem que a gente vá além do que podemos. (ACS 4)

Existe uma relação distorcida quanto ao trabalho da ACS, comunicação zero, apenas cobranças [da equipe de saúde]. (ACS 6)

Apesar das dificuldades com a equipe de saúde da UBS em que atuam, em relação ao relacionamento interpessoal com a chefia imediata, ou seja, com a enfermeira coordenadora do PACS as agentes afirmaram que:

Temos liberdade para desempenhar nosso trabalho, devido a confiança mútua e apoio nas horas difíceis. (ACS 5)

Temos bom relacionamento, diálogo, troca de ideias, flexibilidade e entendimento nas decisões a serem tomadas. (ACS 7)

\section{DISCUSSÃO}

A predominância do sexo feminino na população estudada converge com os resultados de outro estudo realizado com $\mathrm{ACS}^{(10)}$. Identificou-se um grupo de trabalhadoras jovens, com relativa experiência profissional e tempo de convivência com a comunidade em que atuam.
A realização de atividades domésticas reforça a existência da dupla jornada de trabalho das agentes, tendo em vista que esta atividade demanda tempo, esforço físico e envolvimento.

$\mathrm{O}$ fato de exercerem sua atividade de trabalho no mesmo espaço em que vivem pode implicar em maior demanda de tarefas, pois o trabalhador fica a mercê de sua clientela em tempo integral, o que muitas vezes pode traduzir em sobrecarga, dificultando que o trabalhador se "desligue" do trabalho nas suas horas de folga.

Neste estudo, todas as pesquisadas afirmaram gostar do trabalho como ACS. Para que a realização profissional aconteça é essencial "gostar do que faz" e isso pode ser reforçado a partir do aprendizado contínuo, conferindo segurança no cotidiano. Se assim for, é possível que o agente transmita segurança para o usuário, o que pode dar credibilidade ao profissional ${ }^{(11)}$.

No que tange aos hábitos de vida, as pesquisadas afirmaram que não realizam atividades físicas com regularidade, é sabido que o exercício físico pode ser uma forma de lazer e de restaurar a saúde dos efeitos nocivos que a rotina traz ${ }^{(12)}$. Entretanto, destaca-se que diariamente, as agentes realizam caminhadas para as visitas domiciliares $\mathrm{e}$ também atividades domésticas, o que as retira do grupo de pessoas sedentárias. Quanto ao uso de tabaco, uma agente afirmou que fuma, é importante considerar que as demais não utilizam tabaco, o que as protege dos acometimentos oriundos do uso prolongado desse produto.

Quanto à presença de dores nas pernas, coluna vertebral e cansaço entre as ACS, faz-se necessário relacionar estes sintomas com o tipo de atividade exercida, mas também se ressalta a necessidade de identificar medidas para minimizá-las ou anulá-las.

A dor é uma entidade multidimensional, carregando significados inerentes ao mundo do trabalho. Nessa direção, a dor que precisa ser calada reflete a necessidade da pessoa de manter-se sempre apta para o trabalho, íntegra na sua totalidade, produtiva aos olhos dos gestores e das exigências do trabalho. Calar a dor é mimetizar um ideal de trabalhador, constituído para servir ao sistema de produção capitalista ${ }^{(13)}$. Neste sentido, é importante que a dor e o cansaço não sejam banalizados no cotidiano de trabalho das ACS, pois são sinais que necessitam de avaliação e intervenção no sentido de buscar a melhoria da saúde destes trabalhadores.

Parte das ACS reconhece que o trabalho tem influenciado em seu adoecimento, o que é essencial para o enfrentamento deste problema. Pessoas que moram no mesmo bairro ou vizinhança nem sempre têm os mesmos interesses ou se relacionam bem, sendo frequente 
a existência de conflitos, inimizades e disputas ${ }^{(6)}$. Dessa forma, estudo questiona a obrigatoriedade do ACS morar na comunidade onde trabalha, alertando para o fato de isso poder ser fonte adicional de sofrimento psíquico a esses trabalhadores e se tornar patogênico ${ }^{(14)}$.

Com relação às cargas físicas, as condições de trabalho das ACS foram consideradas inadequadas com destaque para o ambiente laboral e a precariedade dos equipamentos de trabalho. Nessa direção, enfatiza-se a necessidade de haver flexibilidade no planejamento das atividades diárias dos agentes, para que as adversidades possam ser trabalhadas e as demandas da comunidade atendidas.

Pesquisa evidenciou que o trabalho dos ACS seria menos desgastante, caso recebessem do poder público materiais simples, como capa de chuva e protetor solar, o que remete aos resultados encontrados neste estudo ${ }^{(15)}$.

Neste contexto, é de suma importância que os gestores dos serviços de saúde se comprometam em fornecer os equipamentos de trabalho necessários aos ACS, conforme a realidade e necessidade dos serviços de saúde, buscando recursos e instrumentalização para estes trabalhadores, no sentido de proporcionar um atendimento de qualidade para os usuários, bem como promoção da saúde para os ACS.

No que tange às cargas psíquicas, identificou-se a falta de reconhecimento, a incapacidade de resolver certos problemas e dificuldade de relacionamento interpessoal.

Quando as demandas da comunidade não são atendidas, esta não reconhece a qualidade das ações do ACS, nem o esforço empregado para realizá-las. Além disso, faz cobranças por uma atuação efetiva e resolutiva que se intensificam pelo constante contato que os usuários mantêm com o agente ${ }^{(14)}$.

O trabalhador deseja ser reconhecido pelo seu trabalho por parte dos demais, o que inclui não somente a equipe da qual faz parte, mas também os usuários do serviço $^{(15)}$. Isso não seria diferente no caso dos ACS que ressaltaram a importância e a satisfação que esta valorização representa em seu cotidiano laboral.

Estudo realizado com ACS de Vitória-ES evidenciou a experiência de gratificação com o trabalho diante do reconhecimento da comunidade, dado também encontrado nesta pesquisa, que pode minimizar os efeitos das cargas psíquicas no trabalho ${ }^{(10)}$.

A carga psíquica diante da incapacidade de resolver certos problemas remete ao fato de que o exercício da profissão de ACS resulta em uma relação com o usuário que, por vezes, apresenta-se permeada de ambiguidades. Os conflitos decorrentes deste processo são fenômenos característicos dessa profissão, podendo causar nos profissionais ansiedade e até incapacidade de apresentar soluções viáveis para a resolução dos problemas vividos pelos usuários ${ }^{(16)}$.

No que tange às dificuldades no relacionamento interpessoal com a comunidade, um estudo explicita que este fato pode acontecer porque a comunidade deposita muitas expectativas nos ACS para a resolução de seus problemas, suscitando dificuldades e tornando os agentes vulneráveis ao sofrimento no trabalho, frente ao não atendimento das demandas. Portanto, é essencial dar suporte profissional aos ACS, para que possam fazer frente a esta situação ${ }^{(15)}$.

No que se refere à equipe de saúde, relações conflituosas entre os ACS e os demais membros da equipe pode implicar em carga de trabalho adicional aos trabalhadores, especialmente quando se trata de equipes grandes e com maior grau de exigência.

Estudo sobre o trabalho em equipe na Estrégia de Saúde da Família evidencia a necessidade de avanços para a consolidação e maior integração da equipe de saúde da família, o que converge com os resultados encontrados neste estudo ${ }^{(17)}$.

A falta de confiança entre os membros da equipe pode trazer conflitos durante o trabalho de modo a comprometer não apenas de forma pontual, mas o relacionamento de toda a equipe e o êxito do trabalho desenvolvido ${ }^{(18)}$, já que os ACS possuem informações importantes que subsidiam o trabalho da enfermeira e dos demais componentes da equipe ${ }^{(19)}$.

Este estudo evidenciou que a maioria dos agentes identifica como ótima a valorização dada pela enfermeira para o seu trabalho. Apoiar, dar subsídios para o trabalho, trocar ideias e conhecimentos são pontos que somam para a prestação de uma assistência qualificada aos usuários. Além disso, é papel do enfermeiro, como líder da equipe de saúde, valorizar esta parceria profissional com as ACS.

As falas das ACS convergem com os resultados encontrados em uma pesquisa em que as enfermeiras que atuam em Estratégia Saúde da Família afirmaram valorizar o trabalho realizado pelos agentes, pelo contato direto que estes mantêm com as famílias ${ }^{(19)}$.

Além das cargas físicas e psíquicas, mencionadas pelos ACS neste estudo, é preciso considerar que o cotidiano laboral destes trabalhadores é permeado por situações complexas, como dinâmicas familiares de difícil intervenção, violência e tráfico de drogas; muitas vezes sem uma rede social instituída e sem a equipe interdisciplinar necessária, propiciando uma demanda emocional que contribui para o esgotamento do profissional ${ }^{(20)}$. 
Assim, evidencia-se que a melhoria das condições e organização do trabalho, a identificação de atribuições de cada trabalhador, a capacitação permanente e a melhoria do processo de comunicação com a equipe de saúde são elementos que podem favorecer a realização do trabalho dos ACS. Além disso, podem minimizar as cargas de trabalho do ACS, o que repercutirá positivamente na saúde desses trabalhadores, bem como na qualidade do trabalho realizado.

\section{CONSIDERAÇÕES FINAIS}

Ao final da pesquisa, pode-se afirmar que o ambiente laboral inadequado e a precariedade dos equipamentos de trabalho foram considerados como cargas físicas no trabalho do ACS.

No que tange às cargas psíquicas, foram mencionadas pelas ACS a falta de reconhecimento por parte de alguns integrantes da comunidade; a incapacidade de resolver certos problemas e a dificuldade de relacionamento interpessoal com a comunidade e equipe de saúde da unidade.

Esse estudo contribuiu para o conhecimento das condições de trabalho das ACS pela equipe de saúde, gestores, sociedade, docentes e estudantes, possibilitando a reflexão de novos meios que proporcionem bem-estar durante o trabalho. Nessa direção, destacase a necessidade de traçar estratégias adequadas de intervenção junto as ACS e ao serviço de saúde, no sentido de promover ações que os despertem para o autocuidado, busca de melhor qualidade de vida, visando favorecer a saúde dos trabalhadores.

Como limitações do estudo apontam-se o fato de ter sido realizado com ACS de uma UBS, o que remete a necessidade de se considerar os resultados deste estudo em sua singularidade, bem como a necessidade de novas investigações sobre as cargas de trabalho dos ACS no município e em outras regiões do país.

Também se recomenda a realização de pesquisas acerca do cotidiano laboral dos ACS que contemplem as facilidades e dificuldades para a realização do trabalho, a fim de traçar estratégias para minimizar os efeitos das cargas de trabalho.

\section{REFERÊNCIAS}

1. Ministério da Saúde (BR). Secretaria Executiva. Programa de Agentes Comunitários de Saúde (PACS). Brasília; 2001.
2. Ministério da Saúde (BR). Secretaria de Atenção Básica. Departamento de Atenção Básica. Política Nacional de Atenção Básica. Brasília; 2006.

3. Nunes MDO, Trad LB, Almeida BDA, Homem CR, Melo MCIDC. O agente comunitário de saúde: construção da identidade desse personagem híbrido e polifônico. Cad. Saúde Pública. 2002;18(6):1639-46.

4. Bezerra AFB, Espírito Santo ACG, Batista Filho M. Concepções e práticas do agente comunitário na atenção à saúde do idoso. Rev. Saúde Pública. 2005;39(5):809-15.

5. Wai MFP, Carvalho AMP. O trabalho do agente comunitário de saúde: fatores de sobrecarga e estratégias de enfrentamento. Rev. enferm. UERJ. 2009;17(4):563-8.

6. Trindade LL, Gonzáles RMB, Beck CLC, Lautert L. Cargas de trabalho entre os agentes comunitários de saúde. Rev. Gaúcha Enferm. 2007;28(4):473-9.

7. Seligmann-Silva E. Desgaste mental no trabalho dominado. Rio de Janeiro: Cortez/UFRJ; 1994.

8. Oliveira RMR. A abordagem das lesões por esforços repetitivos/distúrbios osteomoleculares relacionados ao trabalho - LER/DORT no Centro de Referência em Saúde do Trabalhador do Espírito Santo - CRST/ES. [dissertação]. Rio de Janeiro (RJ): Escola Nacional de Saúde Pública; 2001.

9. Minayo MCS. O desafio do conhecimento: pesquisa qualitativa em saúde. São Paulo: Hucitec; 2007.

10. Barcellos CSN, Pandolfi M, Miotto, MHMB. Perfil do agente comunitário de saúde (ACS) de Vitória - ES. UFES Rev. Odontol. 2006;8(4):21-8.

11. Lima JC, Moura MC. Trabalho atípico e capital social: Os agentes comunitários de saúde na Paraíba. Soc. estado. 2005;20(1):103-33.

12. Silva RS, Silva I, Silva RA, Souza L, Tomas E. Atividade física e qualidade de vida. Ciênc. saúde colet. 2010;15(1):115-20.

13. Neves RF, Nunes MO. Da legitimação a (res) significação: o itinerário terapêutico de trabalhadores com LER/DORT. Ciênc. saúde colet. 2010;15(1):211-20.

14. Jardin TA, Lancman S. Aspectos subjetivos do morar e trabalhar na mesma comunidade: a realidade vivenciada pelo agente comunitário de saúde. Interface-Comunic., Saude, Educ. 2009;13(28):123-35. 
15. Brand CI, Antunes RM, Fontana RT. Satisfações e insatisfações no trabalho do agente comunitário de Saúde. Cogitare enferm. 2010;15(1):40-7.

16. Telles SH, Pimenta AMC. Síndrome de Burnout em agentes comunitários de saúde e estratégias de enfrentamento. Saude soc. 2009;18(3):467-78.

17. Azambuja EP, Fernandes GFM, Kerber NPC, Silveira RS, Silva AL, Gonçalves LHT et al. Significados do trabalho no processo de viver de trabalhadoras de um programa de saúde da família. Texto Contexto Enferm. 2007;16(1):71-9.

18. Valentim IVL, Kruel AJ. A importância da confiança interpessoal para a consolidação do Programa de Saúde da Família. Cienc. saúde colet. 2007;12(3):777-88.

19. Colomé ICS, Lima MADS, Davis R. Visão de enfermeiras sobre as articulações das ações de saúde entre profissionais de equipes de saúde da família. Rev Esc Enferm USP. 2008; 42(2):256-61.

20. Silva ATC, Menezes PR. Esgotamento profissional e transtornos mentais comuns em agentes comunitários de saúde. Rev. Saúde Pública. 2008;42(5):921-9. 